Title:

\title{
Effect of myelin parameters and membrane channel dynamics in the SENN model
}

Authors \& affiliations:

\author{
Thomas Tarnaud ${ }^{1}$, Emmeric Tanghe ${ }^{1}$, Luc Martens ${ }^{1}$, Wout Joseph ${ }^{1}$ \\ ${ }^{1}$ Ghent University, Belgium
}

\begin{abstract}
Introduction
The spatially extended nonlinear node model (SENN, Reilly 1985) is an extension of the McNeal-model (1976), describing electrostimulation of a myelinated neuron. Nonlinear Frankenhaeuser-Huxley equations are used to describe the nodes of Ranvier. In this model the myelin conductance and capacitance is approximated to be zero to save computation time (myelin approximation). To our knowledge, the effect of this approximation on computation time and neuronal excitation parameters has not yet been published. In this study, this topic is investigated, together with the impact on neuronal excitation of substituting the $\mathrm{FH}$-system with other membrane models.
\end{abstract}

\section{Methods}

A Crank-Nicolson discretisation of a $10 \mu \mathrm{m}$ SENN-type neuron (length $\mathrm{L}=3 \mathrm{~cm}$ ) is implemented. Five different versions of the neuron are simulated for a variety of electrode locations and waveform properties. These different versions are obtained by substituting the $\mathrm{FH}$-system with either the Hodgkin-Huxley (HH), Chiu-Ritchie-Rogart-Stagg-Sweeney (CRRSS), SchwarzEikhof (SE) or Schwarz-Reid-Bostock (SRB) membrane-model. Each version of the SENNneuron is simulated twice: first within the approximation of negligible myelin values and second with the myelin properties obtained by Tasaki (1955).

\section{Results}

The effect of the myelin approximation and the membrane-channel model on the excitation parameters of a SENN-neuron is shown in Fig. 1. The myelin approximation reduced the computation time with a mean factor of 2.6. However, it was found that neglecting the myelin capacitance and conductance leads to significant errors when activation at the neuronal terminations contributes to the action potential. E.g. for micro-electrode stimulation at $5 \mathrm{~mm}$, higher errors were observed in anodal $(<13.5 \%)$ than in cathodal strength-duration plots $(<0.55 \%)$.

\section{Discussion}

Neglecting finite myelin parameters shortens simulation time at the expense of computational accuracy. Furthermore, as excitation parameters depend strongly on the membrane-channel model, selection of the appropriate model to describe a specific type of neuron can be accomplished by matching simulated and experimental excitation parameters. 

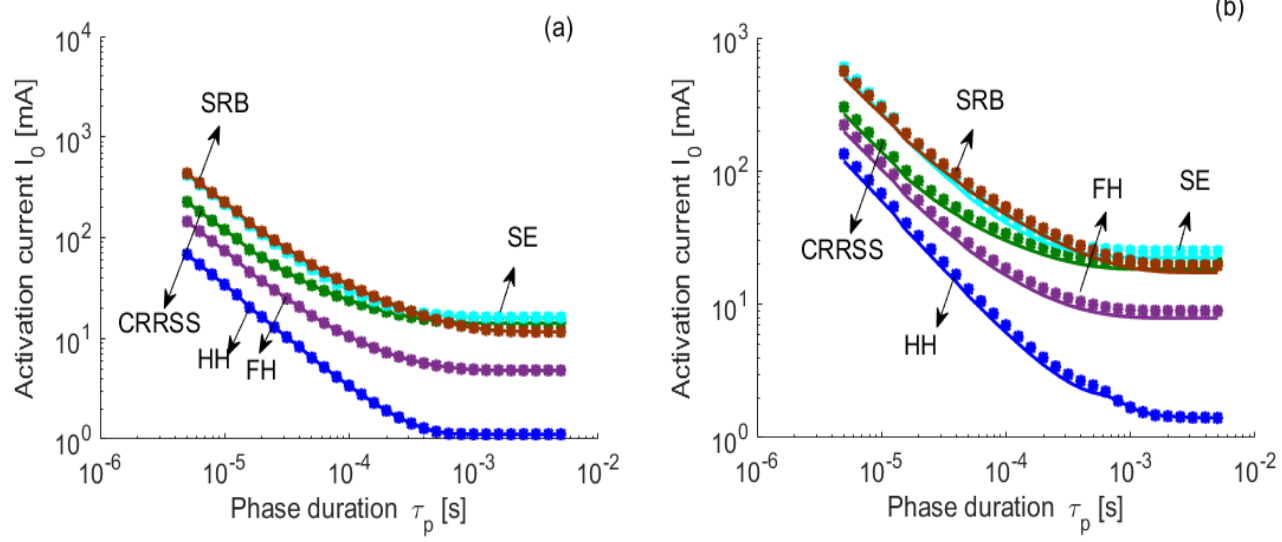

(c)

(d)
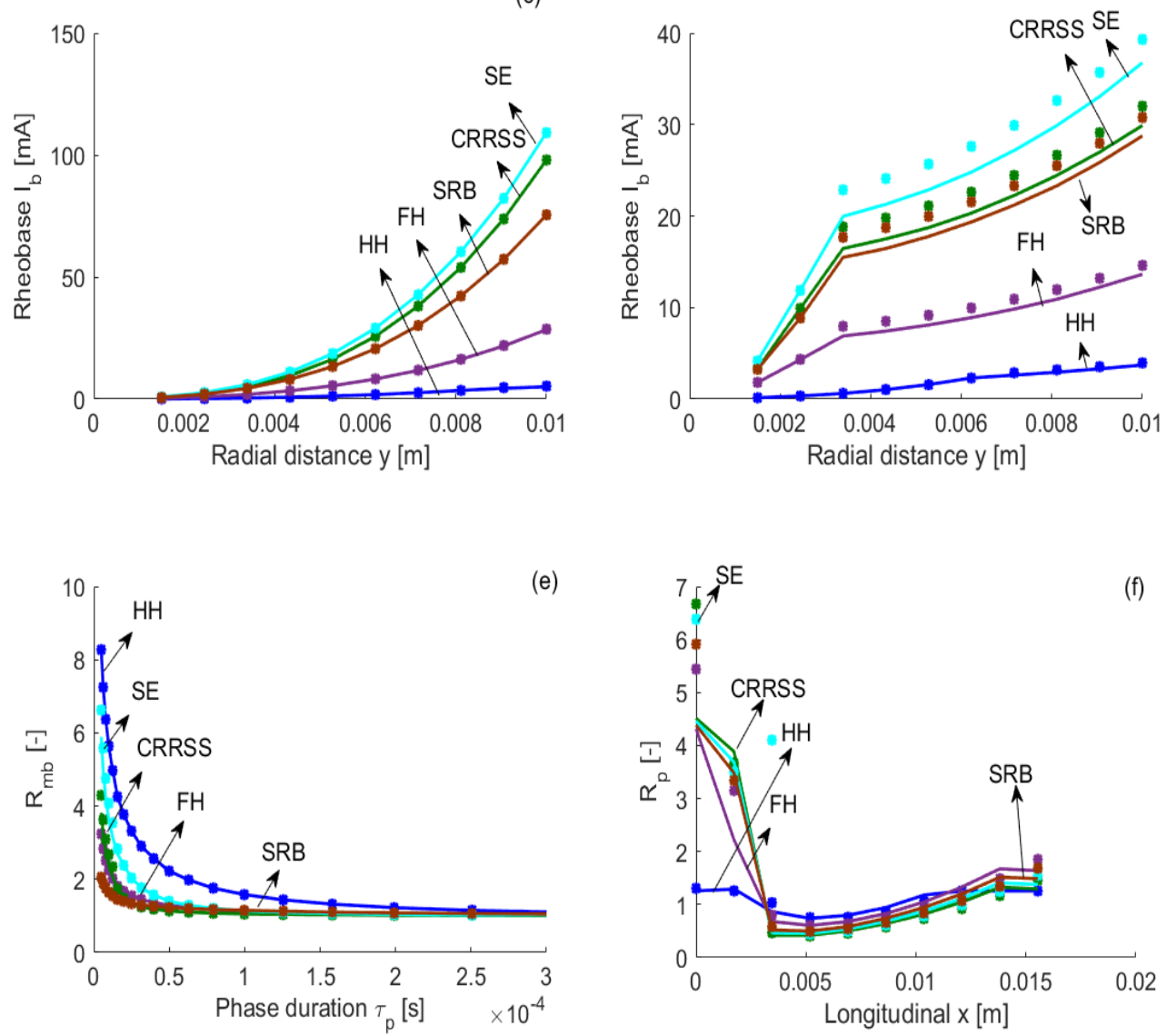

Figure 1. (a) Cathodal strength-duration plot, (b) anodal strength-duration plot, (c) cathodal rheobase, (d) anodal rheobase, (e) cathodal monophasic-biphasic rheobase ratio, (f) polarity ratio. Stars indicate the use of the myelin approximation, while full lines indicate the use of the myelin parameters in Tasaki (1955). 